\title{
PODER, CENTRALIDADE E EMBEDDEDNESS COMO MECANISMOS PARA AUMENTAR A EFICIÊNCIA DE CAPTURA DO CONHECIMENTO DAS SUBSIDIÁRIAS EM SUAS REDES LOCAIS
}

\author{
POWER, CENTRALITY AND EMBEDDEDNESS AS \\ MECHANISMS TO INCREASE SUBSIDIARIES' EFFICIENCY IN \\ GRASPING KNOWLEDGE ON THEIR LOCAL NETWORKS
}

\author{
PODER, CENTRALIDAD Y EMBEDDEDNESS COMO \\ MECANISMOS PARA AUMENTAR LA EFICIENCIA DE \\ CAPTURA DEL CONOCIMIENTO DE LAS SUBSIDIARIAS EN \\ SUS REDES LOCALES
}

\author{
Victor Ragazzi Isaac \\ Professor do Centro Universitário SENAC - Campos \\ do Jordão \\ Doutorando em Administração na Escola Superior de \\ Propaganda e Marketing (ESPM-SP) \\ victor.ragazzi@gmail.com
}

Eduardo Jorge Branco Vieira Barcelos

Professor de Administração nas Faculdades Integradas Rio Branco

Doutorando em Gestão Internacional na Escola Superior de Propaganda e Marketing (ESPM-SP)

eduardobrancobarcelos@gmail.com

\section{Mario Henrique Ogasavara}

Professor de Administração da Escola Superior de Propaganda e Marketing (ESPM-SP)

Pós-doutor na National University of Singapore (Cingapura)

mario.ogasavara@espm.br
Contextus

ISSNe 2178-9258

Organização: Comitê Científico Interinstitucional Editor-Chefe: Diego de Queiroz Machado

Editora Associada: Márcia Zabdiele Moreira Avaliação: double blind review pelo SEER/OJS

Recebido em 01/11/2018 Aceito em 21/03/2019

Versão final em 04/04/2019

http://dx.doi.org/10.19094/contextus.v17i2.39520

\section{RESUMO}

A literatura de Negócios Internacionais enfatiza as relações interorganizacionais em redes de negócios, baseadas no embeddedness, como mecanismo para uma empresa multinacional melhorar seu desempenho. Entretanto, uma análise aprofundada da literatura mostra que o embeddedness por si só é insuficiente para que as subsidiárias obtenham vantagem competitiva local. Assim, buscando compreender melhor esta questão, foi realizada uma revisão sistemática da literatura com 45 artigos que abordam três constructos centrais em redes: embeddedness, poder e centralidade. A análise sugere uma lacuna nos estudos sobre poder e centralidade da subsidiária em sua rede externa. Portanto, por meio de um modelo conceitual, mas com aplicação gerencial, este artigo sugere que a subsidiária utilize de forma integrada embeddedness, poder e centralidade de modo a otimizar o acesso aos recursos tangíveis e intangíveis e reduzir os conflitos da rede local necessários para obter vantagem competitiva específica (SSA) e global (FSA).

Palavras-chave: subsidiária; embeddedness; poder; centralidade; acesso a conhecimento e recursos. 


\begin{abstract}
The International Business literature emphasizes interorganizational relationships in business networks, based on embeddedness, as a mechanism for multinational enterprises to improve their performance. However, an indepth analysis of the literature shows that embeddedness alone is insufficient to subsidiaries to gain local competitive advantage. Thus, in order to better understand this issue, a systematic review of the literature was carried out using the online database with 45 articles that address three central constructs in networks embeddedness, power and centrality. The analysis suggests a gap in the studies on power and centrality of the subsidiary in its external network. Therefore, through a conceptual model, but with managerial application, this article suggests that the subsidiary use embeddedness, power and centrality in an integrated way to optimize access to the tangible and intangible resources, and reduce network conflicts needed to obtain specific (SSA) and global competitive advantage (FSA).
\end{abstract}

Keywords: subsidiary; embeddedness; power; centrality; access to knowledge and resources.

\title{
RESUMEN
}

La literatura de Negocios Internacionales enfatiza las relaciones interorganizacionales en redes de negocios, basadas en el embeddedness, como mecanismo para una empresa multinacional para mejorar su desempeño. Sin embargo, un análisis en profundidad de la literatura muestra que el embeddedness por sí solo es insuficiente para obtener ventaja competitiva local. Así, buscando comprender mejor esta cuestión, se realizó una revisión sistemática de la literatura, utilizando la base de datos online Science Direct. Se seleccionaron 45 artículos que abordan tres constructos centrales en redes (embeddedness, poder y centralidad). El análisis sugiere un gap en los estudios sobre poder y centralidad de la subsidiaria en su red externa. Por lo tanto, a través de un modelo conceptual, pero con aplicación gerencial, este artículo sugiere que la subsidiaria utilice de forma integrada el embeddedness, poder y centralidad para optimizar el acceso a los recursos tangibles e intangibles y reducir conflictos de la red necesarios para obtener ventaja competitiva local (SSA) y global (FSA).

Palabras clave: subsidiaria; embeddedness; poder; centralización; acceso al conocimiento y recursos.

\section{INTRODUÇÃO}

$\mathrm{O}$ atual ambiente dinâmico requer que as empresas multinacionais (MNEs) revejam suas estratégias competitivas em função de mudanças ambientais relevantes que impactam significativamente o seu desempenho. O papel estratégico das subsidiárias (PIASKOWSKA; TROJANOWSKI, 2014), assume destaque quando esta procura capturar e internalizar oportunidades, por meio de mecanismos que garantam acesso ao conhecimento (ANDERSSON; FORSGREN; HOLM, 2007; MEYER; MUDAMBI; NARULA, 2011). Para fazer frente às mudanças ambientais, as MNEs têm implantado mudanças organizacionais, que permitam desenvolver agilidade e novas competências como resposta às variações do ambiente (TEECE; PETERAF; LEIH, 2016).

Kostova, Marano e Tallman (2016) salientam em seu estudo, que nas últimas duas décadas houve um crescente interesse pela teoria de redes de negócios aplicada às MNEs (ANDERSSON; FORSGREN; HOLM, 2002; ZAHEER; GÖZÜBÜYÜK; MILANOV, 2010), utilizando estratégias cooperativas de relacionamento, tanto no nível da empresa através de alianças estratégicas e joint-ventures (PU; SOH, 2018; FERRARIS et al., 2017), como no nível da subsidiária, através da formação de redes locais, entre clientes, fornecedores, universidades e outras instituições de interesse da empresa (MEYER; MUDAMBI; 
PODER, CENTRALIDADE E EMBEDDEDNESS COMO MECANISMOS PARA AUMENTAR A EFICIÊNCIA DE CAPTURA DO CONHECIMENTO DAS SUBSIDIÁRIAS EM SUAS REDES LOCAIS

NARULA, 2011). As conexões cooperativas entre atores facilitam o acesso local ao conhecimento privilegiado e recursos distintivos, que atuam como mecanismos de redução da incerteza ambiental e institucional (CIABUSCHI; DELLESTRAND; HOLM, 2012).

Isso introduz um novo papel à subsidiária que pode ser observado tanto por uma concepção macro, estando vinculado ao país, quanto em uma concepção micro-, se limitando ao ambiente da subsidiária (RUGMAN, 2014). No nível de país, a subsidiária procura desenvolver vantagens especificas (CSAs - country-specific advantages) provenientes de recursos encontrados no país hospedeiro (ANDERSSON; DELLESTRAND; PEDERSEN, 2014). No nível da subsidiária, esta objetiva criar vantagens específicas da subsidiária (SSAs - subsidiary-specific advantages) vinculado ao fortalecimento das relações de interdependência (embeddedness) da sua rede externa, e do desenvolvimento de novas competências organizacionais orquestradas pela MNE, com o fim de gerar vantagens específicas (FSA - firm-specific advantages) da multinational (RUGMAN; VERBEKE; NGUYEN, 2012). Nesse caso, a subsidiária amplia significativamente sua importância como fonte de vantagem competitiva para a MNE (GOVINDARAJAN; RAMAMURTI, 2011).

Gammelgaard et al. (2012) defendem que a subsidiária com autonomia para desenvolver sua rede local e aprofundar seus laços entre os constituintes da rede, pode utilizar o embeddedness como mecanismo de acesso ao conhecimento e recursos distintivos locais. Entretanto, o embeddedness não explica sozinho a seguinte indagação: por que em determinados contextos a subsidiária falha na obtenção do conhecimento e recursos únicos para obter vantagem competitiva? Ou mesmo, há outras variáveis além do embeddedness que possam influenciar a captura de conhecimento e de recursos privilegiados? Baseado nestas duas questões, levanta-se a seguinte pergunta de pesquisa: a subsidiária pode melhorar sua eficiência na captura do conhecimento diferenciado local ao utilizar intencionalmente a centralidade e poder, além do embeddedness, em sua rede externa?

A literatura de Negócios Internacionais tem retratado com muita frequência a questão do embeddedness (KOSTOVA; MARANO; TALLMAN, 2016), tanto na perspectiva relacional, como na estrutural (ANDERSSON; DELLESTRAND; PEDERSEN, 2014). Entretanto, a revisão da literatura sobre centralidade e poder nas subsidiárias mostrou que são raros os estudos (GIGLIO; PUGLIESE; SILVA, 2012), em particular de poder, tanto na literatura de negócios internacionais como na teoria de redes. A teoria de redes apresenta vários estudos sobre a relação positiva entre centralidade estrutural e o poder relacional nas organizações (CHOI; KIM, 2008). Todavia, há discordância quanto a essa relação ser positiva (BONACICH; ROY, 1986). O poder, conforme Provan, Huang e Milward (2009) é 
considerado um fator que afeta o relacionamento entre atores da rede externa impactando a maneira como a rede de negócios se estrutura, porém a ênfase dada em Negócios Internacionais sobre poder recai na rede interna da MNE e o papel de controle e coordenação da matriz (CIABUSCHI; HOLM; MARTIN, 2014).

Este estudo contribuiu para a literatura em três principais aspectos. Primeiro, realizar uma revisão sistemática em uma relação pouco explorada pela literatura de Negócios Internacionais, entre embeddedness, poder e centralidade na rede externa da subsidiária. Segundo, verifica como poder e centralidade podem influenciar ou mesmo melhorar o acesso aos recursos distintivos da rede externa local, que é um dos importantes aspectos na literatura de redes. Terceiro, o uso do construto integrado embeddedness, poder e centralidade pela subsidiária estende a teoria sobre MNEs, e procura dar sustentação teórica para explicar como a eficiência na captura de conhecimento e recursos locais pela subsidiária pode ser efetivamente melhorada. $\mathrm{O}$ estudo ainda possibilita uma contribuição para a prática ao sugerir alguns mecanismos gerenciais de poder e centralidade que reforçam o embeddedness e facilitam a captura de conhecimento local.

Este artigo se estrutura da seguinte maneira: em um primeiro momento é realizado o embasamento teórico, focando nos fatores embeddedness, poder e centralidade. Posteriormente, é apresentada a metodologia de pesquisa, assim como os resultados encontrados. Por fim, é realizada uma análise profunda dos resultados, no qual se é apresentado um modelo conceitual acerca do embeddedness, do poder e da centralidade.

\section{REFERENCIAL TEÓRICO}

Embeddedness, Centralidade e Poder são temas centrais de pesquisa tanto na literatura de Negócios Internacionais como de Redes. Entretanto, o foco em cada uma dessas linhas de pesquisa é diferente, o que abre espaço para utilizar alguns conceitos mais bem desenvolvidos da teoria de redes em Negócios Internacionais. O modelo conceitual foi elaborado conforme diferentes perspectivas dentro da teoria de redes que versão sobre embeddedness, poder e centralidade, que serão abordadas nos próximos subtópicos. 
PODER, CENTRALIDADE E EMBEDDEDNESS COMO MECANISMOS PARA AUMENTAR A EFICIÊNCIA DE CAPTURA DO CONHECIMENTO DAS SUBSIDIÁRIAS EM SUAS REDES LOCAIS

\subsection{Embeddedness na óptica do paradigma social de redes}

Na literatura, o embeddedness tem sido estudado principalmente sob duas vertentes: estrutural e relacional. Na estrutural há a preocupação em se abordar a intensidade dos laços existentes na rede e a posição que cada um dos atores possui dentro da rede, procurando mostrar como esses laços evoluem ao longo do tempo e o impacto de cada ator dentro da rede a partir da sua posição (UZZI; LANCASTER, 2003; KIM, et al., 2014). Em geral temos que os atores centrais da rede, chamados de hubs, são aqueles que possuem um maior acúmulo de conhecimentos e recursos, servindo como atores centrais da rede. Estes atores possuem um maior vínculo de laços com os demais atores da rede. Por outro lado, estes últimos atores são classificados como periféricos, e são definidos como aquelas empresas que possuem uma menor intensidade de laços com os demais atores da rede de negócios, possuindo entretanto, uma maior dependência de recursos por parte dos hubs da rede de negócio em que estão inseridos (CARLEY, 2012; GASPAR, 2014).

Por outro lado, o embeddedness relacional concentra-se em analisar a qualidade dos relacionamentos existentes entre os atores da rede, levando em consideração como os aspectos sociais, tais como confiança, comprometimento e conflito influenciam o relacionamento entre eles. O capital social nos relacionamentos traz benefícios mútuos aos atores da rede de negócio, uma vez que facilita a troca de conhecimento entre os atores, o acesso a recursos únicos e ainda diminui as ações oportunistas graças aos riscos de perda de reputação (MORAN, 2005; LADO; DANT; TEKLEAB, 2008).

Andersson et al. (2002) apontam que o embeddedness relacional é fundamental para o desenvolvimento de competências e capacidades na subsidiária. Nessa perspectiva, Hallin et al. (2011) aponta que o embeddedness relacional com a rede externa local impacta no surgimento de inovações nas subsidiárias de multinacionais. Isso ocorre devido ao acesso a recursos, conhecimentos e aprendizagens compartilhados na rede externa de negócios em que a subsidiária está embedded (BRESCIANI; FERRARIS, 2016). Por outro lado, para se ter o acesso a esses recursos, a subsidiária precisa desenvolver um relacionamento de qualidade, pautado em confiança (DU; WILLIAMS, 2017), comprometimento (HOLM et al., 1999) e adaptação (FERRARIS, 2014), junto aos parceiros locais. Ademais é importante ressaltar que esse processo é de longo prazo, haja vista que a subsidiária, ao entrar em um novo mercado, enfrenta deficiências e vulnerabilidades vinculadas ao liability of foreignness, além do desconhecimento por parte das empresas locais, descrita como liability of outsidership (JOHANSON; VAHLNE, 2009).

12 CONTEXTUS - Revista Contemporânea de Economia e Gestão. Vol. 17 - Nº 2 - mai./ago. 2019 
Por outro lado, é importante ressaltar que estudos (ex. NELL; ANDERSSON, 2012; MASCIARELLI; LAUREN; PRENCIPE, 2010) identificaram que os benefícios do embeddedness podem ser afetados pelo over-embeddedness. Segundo os autores isto pode ser decorrente tanto por questões relacionais, quanto estruturais. As questões relacionais levam em conta os altos investimentos que as subsidiárias realizam para que haja a manutenção dos relacionamentos de qualidade junto aos parceiros locais. Assim, manter um relacionamento baseado em confiança, comprometimento e adaptação é custoso e dispende muito tempo para a subsidiária (MASCIARELLI et al., 2010). Em paralelo, as questões estruturais levam em consideração a complexidade das redes de negócios, apontando que cada parceiro da rede possui uma influência junto à subsidiária, surgindo assim a necessidade de que a subsidiária desenvolva laços (vínculos) mais fortes junto aos principais parceiros, e mais fracos junto aos parceiros emergentes. Isto acaba impactando negativamente no desempenho da subsidiária, pois ela acaba dispendendo recursos escassos (ex. tempo e financeiros), para analisar e compreender esses cenários complexos que as redes de negócios possuem (ANDERSSON et al., 2005; NELL; ANDERSSON, 2012).

\subsection{Poder na óptica da teoria de redes}

Redes interorganizacionais utilizam mecanismos cooperativos de interdependência (OPPER; NEE, 2015), para reduzir a incerteza das transações (DONG et al., 2014). Contudo, assimetrias de informações e conhecimento, conflitos de interesses e variáveis de negociação estão presentes entre os atores da rede, à medida que, mudanças afetam os objetivos individuais e a harmonia das conexões da rede (SHENG-YUE; XU, 2005). Para os objetivos deste artigo o poder interorganizacional é considerado como a capacidade do ator modificar ou controlar comportamentos, atitudes, opiniões, objetivos, necessidades e valores do outro ator (RAHIM, 2016).

Observou-se que a teoria sobre poder interorganizacional evoluiu ao longo do tempo, saindo do nível de transações entre atores e organizações para uma análise dos relacionamentos (REIMANN; KETCHEN, 2017), isto é, pode envolver poder e outros elementos de redes sociais, como: confiança, colaboração e reciprocidade (MEEHAN; WRIGHT, 2012). Das várias tipologias observadas na literatura, duas se destacam: o modelo de French e Raven (1959) tanto utilizado em artigos nacionais (ex. OLIVEIRA et al., 2016) como internacionais (ex. CHAE et al., 2017; REIMANN; KETCHEN, 2017), e o modelo de 
Hardy, Phillips e Clegg (2001). O primeiro modelo é o mais citado pela literatura (MEEHAN; WRIGHT, 2012), razão pela qual foi adotado neste artigo. French e Raven (1959) identificaram cinco fontes de poder entre atores: (1) Recompensa; (2) Coercitiva; (3) Legitimidade; (4) Referência; e (5) Especialista, que serão analisados em profundidade no subtópico 'Poder na óptica de Negócios Internacionais', enquanto, Hardy, Phillips e Clegg (2001) identificaram duas vertentes: (1) Crítica - baseada na posse e posição de recursos, isto é, uma visão de poder por dominação, alinhado com que propõe a teoria de dependência de recursos e o uso do poder coercitivo e punição; (2) Funcionalista - que procura situações propícias para obter mudanças de comportamento dos demais atores, em acordo com as fontes de poder legitimidade, referência e especialista.

O poder pode ser resultado da imposição de regras para que os atores permaneçam na rede (CASTELLS, 2011), o que pode levar a conflitos. Entretanto, outros fatores associados ao dinamismo da rede podem levar alguns atores a procurar ganhar poder ou reduzir de outros (LUHMANN, 2018), devido a mudança de interesses individuais ou a preservação da independência.

Para Nicholls e Huybrechts (2014) e Perer e Shneiderman (2006) o poder está associado à posição formal na rede e controle de recursos e conhecimentos. Ainda que o poder sugira unilateralidade das relações, ele se manifesta principalmente pela interdependência. Dessa maneira, a posse, posição de recursos e acesso ao conhecimento podem ser entendidos também como uma forma de poder (MUDAMBI; NAVARRA, 2015; PROVAN; FISH; SYDOW, 2007).

Ademais, o poder ainda pode ser compreendido em diferentes níveis de análise, assim, no primeiro nível, o da díade, o poder ocorre pela assimetria e dependência que criam laços e restrições de interesse dos atores (ZAHEER; GÖZÜBÜYÜK; MILANOV, 2010). No segundo nível, o do ego ou ator focal, o poder está associado a posição do ator na rede, e no terceiro, a rede em si pode ser vista como um bloco estratégico que busca aumentar o poder de competição.

Por fim, no âmbito de negócios internacionais, o poder é recorrentemente retratado na estrutura da rede interna das multinacionais (FERNER; EDWARDS, 2012). Yamin e Forsgren (2015) apontam que no ambiente da rede interna da MNE, as subsidiárias que possuem uma maior autonomia, apresentam um acesso maior a recursos e conhecimentos estratégicos disponíveis nas redes externas locais em que estão inseridas, desenvolvendo consequentemente um ganho de poder dentro da rede interna da MNE. Por outro lado, o 
acesso a recursos e conhecimentos junto a rede externa local, possibilita o surgimento de inovações de produtos e processos, que posteriormente servirão como barganha de poder para subsidiária dentro da rede da MNE (CIABUSCHI et al., 2012).

\subsection{Centralidade na perspectiva da teoria de redes}

Centralidade usualmente está relacionada ao número de conexões diretas e indiretas que um determinado ator possui (PROVAN et al., 2007; DAS et al., 2018), e mede em algum grau a dependência estrutural entre atores. Em geral, as pesquisas sobre centralidade estão relacionadas ao comportamento ou reputação dos atores, e raramente são voltadas ao acesso ao conhecimento (SHIJAKU et al., 2018; SONG; EVELAND, 2015). Ademais, outras fontes de poder, como atributos pessoais e organizacionais, tais como formação, atividade profissional, prestígio e reputação, e a posição formal do ator dentro da rede, por exemplo, influência, conselhos, suporte, comunicação têm impacto na centralidade da rede e no exercício do poder (TSAI, 2001; MEO et al., 2017; CHANDLER et al., 2013).

Geralmente, a empresa matriz concentra a centralidade e poder dentro da MNE, enquanto as subsidiárias podem ter diferentes níveis de centralidade dependendo da autonomia recebida pela matriz e ao acesso a recursos externos, assim, centralidade é dada pelo grau de dependência relativa entre atores da rede seja ela interna ou externa (DONG $e t$ al., 2015; IBARRA, 1993), cuja dependência de recursos (CAPALDO; GIANNOCCARO, 2014; PARUCHURI, 2010) exerce uma das fontes relevantes de dependência. Entretanto, é a integração destes fatores que traduz melhor o conceito de centralidade na rede (BORGATTI, 2005). Para o constructo centralidade adota-se a proposta de Ibarra (1993), onde centralidade e poder estão interligados, desta maneira, as relações de poder entre atores podem ser intensificadas, dentro de uma perspectiva estrutural, quando determinado ator em posição central na rede tem acesso diferenciado e controle sobre recursos distintivos.

Finalizando esta análise de centralidade em redes, deve-se ainda abordar o conflito como elemento de forte impacto na centralidade, poder e embeddedness. Conflito é definido, neste artigo, como o desequilíbrio no relacionamento entre dois ou mais atores que buscam agir visando somente cumprir seus objetivos (VAALAND; HAKANSSON, 2003). Alguns autores (PANTELI; SOCKALINGAM, 2005; VAHTERA et al. 2017) defendem que algum grau de conflito pode auxiliar na criação e no desenvolvimento de conhecimento, uma vez que os desequilíbrios e problemas entre os atores acabam gerando a necessidade de soluções. Para 
Gadde e Håkansson (1993) o conflito pode ser analisado sob duas perspectivas, a primeira disruptiva e dissociativa, e a segunda ambígua que tanto pode ser benéfica como prejudicial à estabilidade da rede. Nesse segundo caso, o efeito do grau de conflito na eficiência da rede pode ser analisado pela interação de mecanismos de cooperação e de conflito. Como resultado é possível obter alta eficiência da rede, com baixo grau de conflito e alta cooperação, como também com alta cooperação e alto conflito, pois elementos mitigadores são acionados, como regras, normas e mecanismos institucionais no nível da rede, ou, a aceitação da posição de poder de determinado ator, por meio de instrumentos de recompensa, coerção, especialização, referência e legitimidade (PROVAN et al., 2008) no nível das díades.

Em outras palavras, ao utilizar adequadamente a centralidade e as fontes de poder, a subsidiária pode melhorar seu acesso ao conhecimento e a recursos da rede externa, mitigando conflitos e contribuindo para aumentar a eficiência da rede como um todo.

\section{METODOLOGIA}

Como método de análise foi adotada a revisão sistemática da literatura, que ajuda a responder a algumas questões ainda não tratadas pela academia e permite ainda identificar, avaliar e sintetizar a literatura relevante sobre determinado tema (PETTICREW; ROBERTS, 2008, p. 9), diferentemente, da revisão tradicional ou a narrativa. Com isto, esta pesquisa caracteriza-se como exploratória e descritiva, ao passo que propõe um modelo conceitual original, formulado a partir de proposições desenvolvidas a partir da revisão sistemática da literatura (CONGALVES, 2005). Ademais, este artigo é de natureza mista, uma vez que se utiliza de estratégias quantitativas e qualitativas na análise dos dados encontrados.

Quanto ao procedimento metodológico, ressaltamos que foram realizadas pesquisas nas bases de dados online Google Academic e Science Direct, devido aos portais oferecerem uma extensa quantidade de periódicos de alta qualidade e que são referência na área de Negócios e Negócios Internacionais. O período da pesquisa se deu de 2000 a 2017, intervalo este com maior concentração de publicações sobre os construtos embeddedness, centralidade e poder em Negócios Internacionais. Para análise dos mesmos construtos na teoria de redes, alguns artigos anteriores a esse período foram analisados por corresponderem à artigos seminais e de maior debate acadêmico. Foram utilizados os seguintes descritores: "embeddedness subsidiary external environment network"; "subsidiary power external 
environment network"; "subsidiary centrality external environment network". Destacamos também que foram contemplados na pesquisa somente periódicos classificados como A1 pelo sistema Qualis da CAPES (International Business Review; Journal of World Business; Journal of International Management; Management International Review; Journal of International Business Studies; e Long Range Planning). Do total de artigos encontrados, 45 foram selecionados. Esta seleção se deu pela leitura do abstract dos artigos encontrados, tendo sido selecionados e analisados os artigos que retratassem sobre: $i$ ) o impacto da rede externa no desempenho da subsidiária; ii) aspectos de poder na rede externa em que a subsidiária está inserida; iii) aspectos de centralidade na rede em que a subsidiária está inserida, e $i v$ ) interação entre poder e centralidade na rede como fonte diferenciada de recursos.

Ademais, ressaltamos que por meio da análise em profundidade da revisão sistemática da literatura desenvolvemos um conjunto de proposições, que serviram de base para a criação do modelo conceitual. Em adição, para elaboração destas proposições, também foram utilizados modelos de artigos anteriores ao período analisado, e que são considerados seminais na área de Redes de Negócios. Esses modelos são oriundos de French e Raven (1959), Raven (1965, 1993), Ibarra (1993). Assim, foram apresentadas quatorze proposições que embasaram o modelo teórico por nós proposto. Deste total, duas estão vinculadas a embeddedness, onze vinculadas a poder e uma a centralidade. Evidenciamos também que o modelo conceitual tem como escopo aglutinar as três variáveis por nós estudadas, podendo ser utilizado em futuros artigos de pesquisa empírica para explicação de fenômenos vinculados a subsidiárias e ganhos de vantagens competitivas.

\section{ANÁLISE E DISCUSSÃO DOS RESULTADOS}

Nesta seção serão discutidos os resultados encontrados na revisão sistemática da literatura, abordando a análise temporal das publicações e os principais autores e periódicos que abordaram sobre a temática. Também iremos contemplar o desenvolvimento das proposições a partir das pesquisas analisadas e, por fim, a elaboração do modelo conceitual teórico, embasado pelas preposições. 
PODER, CENTRALIDADE E EMBEDDEDNESS COMO MECANISMOS PARA AUMENTAR A EFICIÊNCIA DE CAPTURA DO CONHECIMENTO DAS SUBSIDIÁRIAS EM SUAS REDES LOCAIS

\subsection{Análise temporal}

Conforme apontado na seção de metodologia, 45 artigos foram analisados durante o período de 2000 a 2017. Deste total, 56\% concentram-se nos últimos cinco anos (Figura 1), havendo uma crescente utilização das variáveis embeddedness, poder e centralidade para explicar os fenômenos internos das subsidiárias

Figura 1 - Distribuição anual

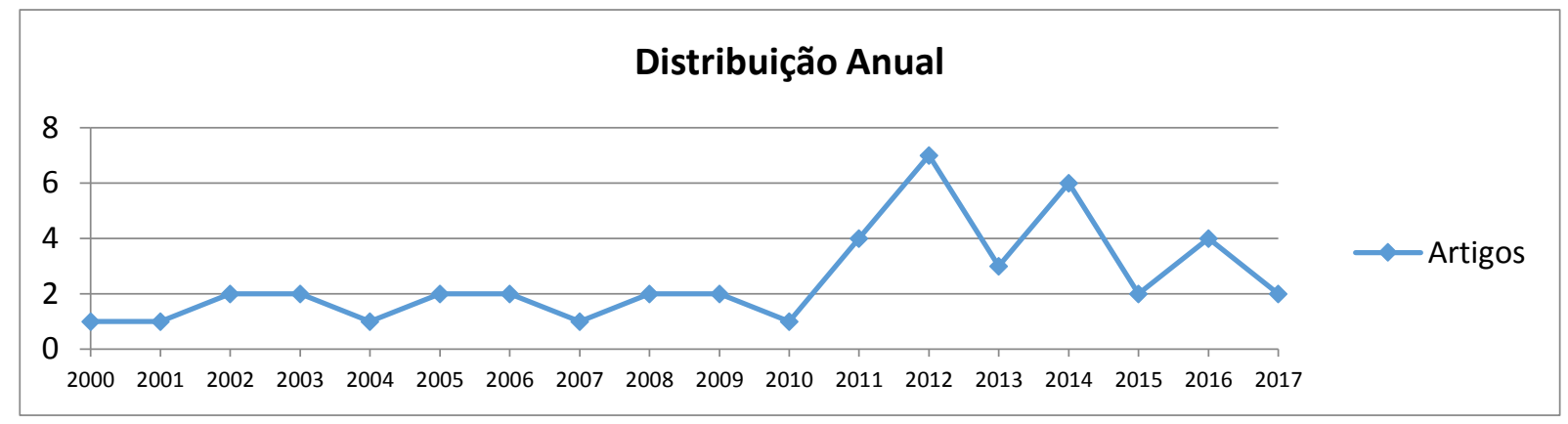

Fonte: elaboração própria.

Ademais, nota-se que entre 2012 e 2014 há um significativo aumento de publicações envolvendo as variáveis analisadas, concentrando se na área negócios internacionais e estando provavelmente associado com o crescente interesse acadêmico sobre como as subsidiárias podem aumentar sua eficiência na captura de conhecimentos e recursos externos, com foco nos mecanismos que podem influenciar neste processo.

\subsection{Principais autores e periódicos}

A maior parte dos artigos se concentrou em analisar o papel do embeddedness no desempenho da subsidiária da MNE e no acesso ao conhecimento e recursos distintivos da rede externa. Destaca-se aqui, que os autores com mais publicações foram Andersson (2000, 2001, 2002, 2007, 2010, 2014); Holm (2001, 2002, 2007, 2010, 2012, 2014) e Forsgren (2000, 2001, 2002, 2007). Adicionalmente, foram encontrados sete artigos sobre poder, redes e subsidiárias, onde se destacam Gammelgaard e Dörrenbächer (2009, 2012, 2016). Somente um artigo (TEIGLAND; WASKO, 2009), sobre centralidade, rede e subsidiária foi encontrado, que reflete a necessidade de se desenvolver trabalhos de centralidade na rede em relação a subsidiária. 
Também foi constatado que os principais periódicos que publicam sobre o tema pesquisado são IBR - International Business Review, com 14 publicações e o MIR Management International Review, com 7 publicações. Importante ressaltar que a equipe editorial do IBR é composta de alguns autores importantes para área de redes e negócios internacionais, como o próprio Andersson, que aparece como autor com maior número de publicações.

\subsection{Análise em profundidade da revisão sistemática e proposta do modelo conceitual}

Esta subseção está dividida em três (3) partes. As duas primeiras retrataram sobre as teorias que envolvem embeddedness, poder e centralidade, na perspectiva de Negócios Internacionais. A terceira, por fim, trata do modelo conceitual e das proposições associadas.

\subsubsection{Embeddedness na óptica das MNEs}

Em relação aos estudos de subsidiárias, dois modelos de embeddedness relacional são utilizados para explicar a captura do conhecimento e o surgimento de inovação local (KOSTOVA; MARANO; TALLMAN, 2016). São eles, o heterárquico (HEDLUND, 2016) e o metanacional, proposto por Doz, Santos e Williamson (2001), que consideram a subsidiária imbricada na rede externa influenciada pelo relacionamento entre os atores locais, como distribuidores, clientes e fornecedores.

O modelo heterárquico (HEDLUND, 2016) sugere que as subsidiárias com maior autonomia perante a matriz apresentam um maior relacionamento com os atores que compõem a rede local externa. A subsidiária utiliza-se das conexões locais e se apropria dos benefícios do embeddedness relacional, como a troca de informações, troca de recursos humanos e tecnológicos, que facilitam o acesso ao conhecimento da rede e possibilitam criar competências (ZANDER; MATHEWS, 2010; ISAAC et al., 2018).

Complementando o modelo heterárquico, Doz, Santos e Williamson (2001) projetam através do modelo metanacional, que deve haver um alto grau de autonomia das subsidiárias em relação à matriz, fazendo com que a subsidiária assuma a capacidade de realizar determinadas tarefas desenvolvendo novas e distintivas competências e obter vantagem competitiva no país em que atua (GOVINDARAJAN; RAMAMURTI, 2011). Evidencia-se 
PODER, CENTRALIDADE E EMBEDDEDNESS COMO MECANISMOS PARA AUMENTAR A EFICIÊNCIA DE CAPTURA DO CONHECIMENTO DAS SUBSIDIÁRIAS EM SUAS REDES LOCAIS

que tanto no modelo metanacional quanto no modelo heterárquico, o embeddedness relacional pode explicar a captura de informações, conhecimento e oportunidades transformadas em vantagens específicas locais (SSAs) pela subsidiária (MEYER; MUDAMBI; NARULA, 2011; ÁLVAREZ; CANTWELL, 2011).

Entretanto, para que o embeddedness seja eficiente deve haver o aproveitamento dos nós formados dentro da rede os quais dar-se-ão a partir do momento em que a subsidiária apresente a habilidade de reconhecer o valor das informações e conhecimentos trocados com os demais atores (TEECE, 2016), para poder, posteriormente, internalizá-las criando novos produtos, processos e desenvolvendo áreas como P\&D, marketing e gestão (CANTWELL; MUDAMBI, 2005). Em outras palavras, o novo conhecimento gerado pela subsidiária e sua rede externa poderá ser transformado em vantagem local da subsidiária (SSA/LB - local bound), assim como, o conhecimento global poderá criar vantagens para a MNE (FSA/NLB non local bound). Em outras palavras, a subsidiária ao integrar o conhecimento da rede externa e o da rede interna poderá eventualmente, transferir esse conhecimento em âmbito global, na matriz e nas outras subsidiárias (ÁLVAREZ; CANTWELL, 2011; ANDERSSON; FORSGREN; HOLM, 2002), o que é conhecido como embeddedness múltiplo (MEYER; MUDAMBI; NARULA, 2011).

O embeddedness pode ser medido pelo grau de interdependência entre atores, isto é, quanto maior a interdependência entre subsidiária e constituintes da rede, maior o embeddedness da subsidiária na rede (ANDERSSON; FORSGREN, 2000). Outros fatores como confiança e comprometimento influenciam o embeddedness. Assim, embeddedness é influenciado pela confiança, pois quanto maior a confiança entre os atores da rede, maior será a troca de informações e o acesso a recursos únicos (SCHOEMAKER; DAY; SNYDER, 2013). O comprometimento, por seu lado, ressalta a importância das relações entre atores, assim, quanto maior for o comprometimento por parte do ator, maior serão os investimentos realizados pelo mesmo para realizar adaptações ou mudanças em seus produtos ou processos, com o propósito de beneficiar todos os parceiros da rede de negócios (CHANG; CHIANG; PAI, 2012).

Destaque-se que alguns autores (MOLINA-MORALES; MARTÍNEZ-FERNÁNDEZ, 2009; MASKELL; BATHELT; MALMBERG, 2006; BATHELT; MALMBERG; MASKELL, 2004) observaram que o forte aumento na intensidade das relações de interdependência ou over-embeddedness (relacional e estrutural), ao ocorrer em algum momento da existência da rede, pode impactar negativamente os benefícios do embeddedness. 
Diferentes razões levam ao over-embeddedness: excessiva dependência entre alguns atores; proximidade cognitiva, organizacional e social; grande número de laços redundantes e excesso de normas e restrições. Estes fatores limitam o acesso a novas fontes de conhecimento, devido à incerteza na transação, envolve custos adicionais e, por isso, os atores tendem a manter as relações existentes (MASCIARELLI; LAURSEN; PRENCIPE, 2010), reduzindo significativamente as oportunidades fora da rede local.

A partir destes argumentos, as proposições 1 e 2, apresentadas na figura 2, representam este corpo teórico:

Proposição 1: O embeddedness da rede externa da subsidiária está positivamente associado ao acesso ao conhecimento e recursos da rede e fora da rede.

Proposição 2: Over-embeddedness da rede externa da subsidiária está negativamente relacionado ao acesso ao conhecimento e recursos fora da rede.

\subsubsection{Poder na óptica de Negócios Internacionais}

A rara literatura sobre poder em Negócios Internacionais analisa as relações de poder entre matriz e subsidiárias (rede interna da MNE), e raramente as relações de poder entre subsidiárias e suas redes externas, como observado nos trabalhos de Heidenreich (2012) e Andersson, Forsgren e Holm (2007). Exemplificando a relação entre subsidiária e matriz (rede interna), Bouquet e Birkinshaw (2008) investigaram como os atores da rede interna de uma MNE, em posições fracas podem aumentar seu poder dentro da MNE, procurando por legitimidade, acesso a recursos diferenciados e centralidade. Dörrenbächer e Gammelgaard (2016) e Gammelgaard (2009) estudaram como a iniciativa empreendedora da subsidiária pode chamar a atenção da matriz, aumentando seu poder relativo dentro da MNE. Complementando, Gammelgaard et al. (2012) estudaram a rede interna da subsidiária e observaram que o poder obtido pela autonomia da subsidiária, facilita o desenvolvimento da sua rede externa, assim como melhora o desempenho da MNE. Para maiores detalhes sobre a evolução da relação matriz-subsidiária, vide o artigo de Kostova, Marano e Tallman (2016), que analisam as contribuições dos últimos 50 anos do Journal of World Business.

De um modo geral, os estudos de poder em Negócios Internacionais estão concentrados nas teorias da dependência de recursos (CAPALDO; GIANNOCCARO, 2014; PARUCHURI, 2010) e micropolítica (DÖRRENBÄCHER; GAMMELGAARD, 2016), algo 
PODER, CENTRALIDADE E EMBEDDEDNESS COMO MECANISMOS PARA AUMENTAR A EFICIÊNCIA DE CAPTURA DO CONHECIMENTO DAS SUBSIDIÁRIAS EM SUAS REDES LOCAIS

similar ao observado na revisão da literatura das relações de poder em redes interorganizacionais. Além disso, observou-se que o embeddedness da subsidiária em sua rede externa é o mecanismo de maior relevância de acesso ao conhecimento local (MEYER; MUDAMBI; NARULA, 2011). Este por sua vez, aumenta o poder da subsidiária dentro da MNE (DJODAT, 2016), reduzindo o poder relativo da matriz (DÖRRENBÄCHER; GEPPERT, 2013; GEPPERT; DÖRRENB ÄCHER, 2014).

Deve-se ressaltar que, segundo Oliveira et al. (2016), em redes interorganizacionais a dependência de recursos entre atores é o elemento central de poder na rede e pode se sobrepor as relações entre atores. A posse de recursos diferenciados, como tecnologia, pode levar ao desequilíbrio de poder na rede externa, com o consequente aumento de poder relativo (MUDAMBI; PEDERSEN; ANDERSSON, 2014).

A utilização de conceitos de micropolítica em Negócios Internacionais é recente e ainda pouco explorada (DÖRRENBÄCHER; GEPPERT, 2013), e geralmente está associada às relações de poder nas MNEs. Adota-se a definição de micropolítica como as estratégias que os atores utilizam para negociar seus interesses na rede com o fim de ganhar e exercer poder e influência (JANNECK; STAAR, 2010). A micropolítica geralmente é investigada por outras abordagens como a sociológica, a teoria da agência e neoinstitucionalismo, mas, alguns aspectos relevantes das relações de poder e micropolítica podem ser estendidos à MNE e aplicados à rede externa da subsidiária. Por exemplo, na abordagem micropolítica, sob a perspectiva de poder episódico, os atores da rede procuram a posse de recursos para utilizá-los em seu interesse em diferentes contextos, enquanto na perspectiva do poder sistêmico, regras informais ou institucionalizadas da rede limitam o poder (MORGAN; KRISTENSEN, 2006; PROVAN et al., 2008).

Alguns autores consideram o poder prejudicial à qualidade das relações interorganizacionais (NAUDÉ; BUTTLE, 2000) ou ao desenvolvimento da confiança, enquanto outros defendem que o poder é o elemento central da rede por ser um mecanismo de coordenação e cooperação (BACHAMANN; ZAHEER, 2006). Em suma, a eficiência da rede depende tanto do grau de embeddedness da rede, como do uso adequado do poder e da centralidade. Estes argumentos suportam a proposta deste artigo sobre a relevância do embeddedness, poder e centralidade como elementos que combinados podem intensificar a captura de oportunidades, conhecimento, recursos locais e, torná-los vantagens especificas locais ou mesmo globais (RUGMAN; VERBEKE, 2001). Portanto é importante analisar as fontes potenciais de poder, premissa deste estudo que analisa o poder organizacional e o individual (DRENKARD, 2012; RODRIGUEZ et al., 2007) e a centralidade como fonte de 
poder (GOMÉZ, 2003), isto é, a posição privilegiada na rede, permite maior acesso a recursos e informações.

\subsubsection{Modelo Conceitual}

Uma vez estabelecidas as bases teóricas deste estudo quanto a embeddedness, poder e centralidade foi proposto um modelo conceitual que integra esses constructos visando maximizar o acesso a conhecimentos e recursos locais e minimizar o efeito de algumas das dimensões de influência negativa de poder e do over-embeddedness. A base do modelo conceitual proposto é o modelo de French e Raven (1959) uma das tipologias mais utilizadas e adotadas sobre poder (ex. SOMECH; DRACH-ZAHAVY, 2002), atualizado por Raven (1965) e Raven (1993).

As cinco bases do poder (FRENCH; RAVEN, 1959), que levam a mudanças de comportamento dos atores da rede, são: (1) Coercitiva (C) - capacidade de punir outros atores por desvio das normas e regras da rede; (2) Recompensa (Rw) - capacidade de recompensar ou intermediar benefícios para atores da rede por agirem dentro das normas e regras; (3) Legitimidade $(\mathrm{L})$ - direito e autoridade formal associado à posição ou hierarquia na rede, que permite aplicar demandas e envolve obediência; (4) Especialista (E) - refere-se à posse, geralmente individual, de conhecimento, perícia ou competências, e (5) Referência (Re) capacidade individual que leva a admiração, respeito, carisma pelos outros atores da rede. Posteriormente, Raven (1965) inclui no modelo original de 1959 uma sexta base de poder, o (6) poder da informação (I) - capacidade individual ou organizacional que envolve a posse de informações que se traduzem em mudanças na rede, que se sobrepõe em parte ao poder especialista. Raven (1993) estendeu o entendimento do modelo de French e Raven (1959) e Raven (1965) argumentando que cada uma das seis bases de poder, podem apresentar outras dimensões. Assim, coerção (C) e recompensa (Rw) podem ser classificadas como impessoal (a recompensa em si) ou pessoal (quando há a aprovação de determinado indivíduo); legitimidade (L) se divide em quatro dimensões: hierarquia ou posição (Lp), reciprocidade (Lr), equidade (leq) e dependência (Ld), que são autoexplicativas; especialista (E) e referência (Re) podem ser tanto positivas ou benéficas (Ep, Rep), quando aceitas pelos atores ou negativas (En, Ren), quando rejeitadas, e estão relacionadas à variação do contexto da rede como clima interorganizacional e grau de conflito; e informação (I) que pode ser transmitida direta ou indiretamente. 
PODER, CENTRALIDADE E EMBEDDEDNESS COMO MECANISMOS PARA AUMENTAR A EFICIÊNCIA DE CAPTURA DO CONHECIMENTO DAS SUBSIDIÁRIAS EM SUAS REDES LOCAIS

Quanto à relação entre as dimensões de poder e acesso ao novo conhecimento ressaltese que o poder coercitivo é negativamente associado ao acesso e ao conhecimento (RAHIM; MAGNER; ANTONIONI; RAHMAN, 2001) devendo ser evitado, em qualquer contexto, pela subsidiária. Assim como, em situações de conflito, a legitimidade formal e de dependência são negativas e devem ser evitadas, devido ao seu caráter impositivo, enquanto, a legitimidade recíproca e por equidade são positivas e devem ser incentivadas dentro da rede. O poder especialista e referência é contingencial, assim, quando aceitos pela rede devem ser incentivados, pelo seu efeito agregador e de coordenação, entretanto, em contexto de conflitos eles podem ser contestados por alguns atores da rede, e devem ser utilizados cuidadosamente. De um modo geral, o poder do especialista e referência é bem aceito pelos atores da rede, e têm menor impacto que as dimensões coerção, legitimidade legal e de dependência. O poder da informação (I), seja indireto ou direto, é positivo e deve ser estimulado como forma da subsidiária aumentar seu acesso ao conhecimento distintivo.

As proposições três (3) a nove (9) referem-se a poder e centralidade e estão contidas no modelo conceitual da Figura 2. As proposições um (1) e dois (2) relacionadas a embeddedness e over-embeddedness se integram ao modelo, destacando que o embeddedness é a principal fonte de conhecimento e recurso externo. O modelo considera que a dinâmica da rede externa da subsidiária tende a levar a conflitos, seja por interesses individuais ou por mudanças na posse de recursos, que afetam o equilíbrio da rede e a capacidade de a subsidiária acessar conhecimento e recursos locais. Portanto:

Proposição 3: o poder coercitivo está associado negativamente ao acesso de conhecimento e recursos da rede externa da subsidiária.

Proposição 4: o poder por recompensa que beneficia os atores da rede está associado positivamente ao acesso de conhecimento e recursos da rede externa da subsidiária.

Proposição 5a: o poder por legitimidade pela posição na rede, em condições de conflito, está associado negativamente ao acesso de conhecimento e recursos da rede externa da subsidiária.

Proposição 5b: o poder por legitimidade pela reciprocidade mútua está associado positivamente ao acesso de conhecimento e recursos da rede externa da subsidiária.

Proposição 5c: o poder por legitimidade pela equidade está associado positivamente ao acesso de conhecimento e recursos da rede externa da subsidiária.

Proposição 5d: o poder por legitimidade pela dependência está associado negativamente ao acesso de conhecimento e recursos da rede externa da subsidiária. 
Proposição 6a: o poder especialista, com a aprovação dos atores da rede, está relacionado positivamente ao acesso de conhecimento e recursos da rede externa da subsidiária.

Proposição 6b: o poder especialista, rejeitado pelos atores da rede, está relacionado negativamente ao acesso de conhecimento e recursos da rede externa da subsidiária.

Proposição 7a: o poder por referência, com a aprovação dos atores da rede, está associado positivamente ao acesso de conhecimento e recursos da rede externa da subsidiária.

Proposição 7b: o poder por referência, rejeitado pelos atores da rede, está associado positivamente ao acesso de conhecimento e recursos da rede externa da subsidiária.

Proposição 8: o poder por influência está relacionado positivamente ao acesso de conhecimento e recursos da rede externa da subsidiária.

Proposição 9: a centralidade como fonte de poder está relacionada positivamente ao acesso de conhecimento e recursos da rede externa da subsidiária.

Figura 2 - Modelo conceitual de embeddedness, poder e centralidade no acesso a conhecimento e recursos na rede externa da subsidiária

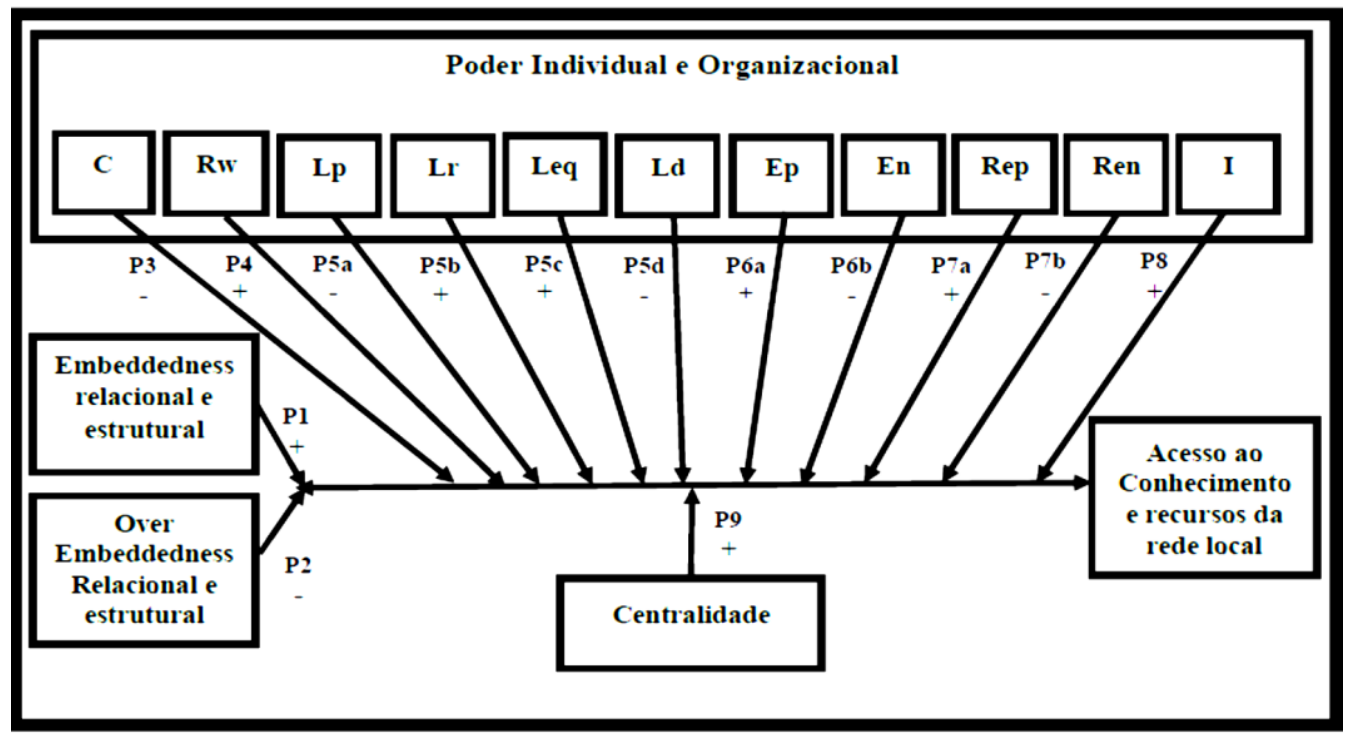

Tipos de Poder Individual e Organizacional: C - coercitiva; Rw - recompensa; Lp - posição; Lr reciprocidade; Leq - equidade; Ld - dependência; Ep - especialista; Em - Rep - referência (+); Ren referência (-); I - influência

Fonte: baseado em French e Raven (1959), Raven (1965, 1993), Ibarra (1993), Ferraris et al. (2017) e Nell e Andersson (2012).

Ponderamos que o modelo conceitual integra as dimensões de embeddedness, poder e centralidade que positivamente e negativamente influenciam o acesso a recursos e conhecimentos externo. O modelo foi elaborado nos pressupostos dos conceitos de poder contidos nas tipologias de French e Raven (1959), Raven (1965) e Raven (1993), de 
centralidade da subsidiária na rede local como fonte de poder de Ibarra (1993) e do embeddedness de Ferraris et al. (2017) e de Nell e Andersson (2012) como principal mecanismo de acesso a recursos locais e conhecimentos, ressaltando-se que o overembeddedness pode influenciar negativamente a captura de conhecimento, como sugerido por Nell e Andersson (2012). Como resultado, esses constructos combinados buscam a maior eficiência da rede utilizando estratégias competitivas que procuram alto grau de embeddedness independente de contextos de baixo conflito e alta cooperação ou de alto conflito e alta cooperação, conforme proposto por Gadde e Håkansson (1993, p. 75) e Gilsing et al. (2008).

\section{CONSIDERAÇÕES FINAIS}

A revisão sistemática da literatura mostrou que poder e centralidade da subsidiária em sua rede externa são raramente estudados em Negócios Internacionais, pois os poucos trabalhos observados se atêm à rede interna da MNE (CIABUSCHI; HOLM; MARTIN, 2014). Entretanto, poder e centralidade são mecanismos importantes de acesso ao conhecimento e recursos privilegiados da rede local, devido a influência, em geral positiva, da posição diferenciada obtida por um ator através do poder e centralidade (DÖRRENBÄCHER; GAMMELGAARD, 2016; GAMMELGAARD et al., 2012; DONG et al., 2015). A literatura de Negócios Internacionais tem dado relevância às MNEs em redes, com foco nas relações de interdependência (embeddedness) tanto da rede interna como externa da subsidiária, e como capturar novo conhecimento, que pode ser transformado em vantagem competitiva (RUGMAN, 2014), específica da subsidiária (SSA), e eventualmente em vantagem específica da MNE (FSA). Da análise conduzida, argumenta-se que embeddedness, poder e centralidade na rede são construtos centrais que podem ser integrados, pois atuam no nível da rede, permitindo que a subsidiária aumente sua eficiência no acesso ao conhecimento e recursos distintivos da rede externa.

O embeddedness relacional está associado com a qualidade das relações de confiança, comprometimento e conflito entre atores e pode reduzir a incerteza e o custo das transações na rede (DONG et al., 2014), como também facilitar o acesso ao conhecimento da rede. De forma semelhante, o poder relacional é fator que influência as relações de confiança, comprometimento e de conflito (PROVAN; HUANG; MILWARD, 2009), o que sugere que embeddedness e poder podem ser utilizados em conjunto para melhorar as relações entre 
atores, assim como mitigar conflitos. Entretanto, é fundamental que a subsidiária evite o overembeddedness, pois mesmo este fortalecendo os laços entre atores da rede, a captura de novas oportunidades é comprometida pela maior incerteza de estabelecer trocas fora da rede, pelo maior custo envolvido e pelas normas que restringem ações externas à rede. Este contexto é crítico em ambientes dinâmicos, onde a construção do novo conhecimento e de novas competências são necessárias para que a subsidiária se adapte adequadamente às mudanças de ambiente.

Poder relacional e a centralidade estrutural, através de suas dimensões: posição na rede, coerção, recompensa, legitimidade, conhecimento especialista, referência e informação facilitam o acesso da subsidiária ao conhecimento diferenciado e privilegiado (BORGATTI, 2005) e potencializam seu efeito na rede quando integrados (IBARRA, 1993). Contudo, em ambientes dinâmicos, a rede pode gerar conflitos entre alguns dos seus atores, devido a mudanças dos interesses individuais e organizacionais (LUHMANN, 2018), ou mudanças no acesso a recursos específicos. Neste contexto de mudança, a subsidiária deve estrategicamente monitorar tanto o ambiente (TEECE; PETERAF; LEIH, 2016) como a rede e estabelecer quando utilizar os mecanismos que melhoram o acesso ao conhecimento (BACHAMANN; ZAHEER, 2006), ou evitar a utilização daqueles que prejudicam as relações interorganizacionais (NAUDÉ; BUTTLE, 2000), conforme proposto no modelo conceitual na Figura 2.

Como contribuição teórica, este estudo estende a literatura de Negócios Internacionais ao propor o uso do embeddedness, poder e centralidade integrados na rede externa da subsidiária como meio de aumentar sua eficiência na captura ao novo conhecimento, mesmo em ambientes de mudança e conflitos. A questão de pesquisa é respondida à medida que foram encontrados argumentos que possibilitam a integração e a interação dos constructos embeddedness, poder e centralidade no nível da subsidiária e da rede local.

Como contribuição gerencial propõe-se que os gestores apliquem os conceitos relacionados às proposições, ao modelo conceitual e ao monitoramento do ambiente e da rede na elaboração das suas ações estratégicas. Por exemplo, a subsidiária pode fortalecer sua posição na rede e melhorar seu acesso ao conhecimento e recursos únicos através do uso adequado das informações monitoradas do ambiente e da rede, na perspectiva do modelo conceitual. Estas informações fornecem aos gestores da subsidiária os mecanismos que orientam suas ações de poder e centralidade, como compartilhamento de benefícios, tornar-se referência da rede, a procura de legitimidade e de liderança da rede, melhorar sua posição na rede, além de atuar na melhoria das relações de interdependência. 
PODER, CENTRALIDADE E EMBEDDEDNESS COMO MECANISMOS PARA AUMENTAR A EFICIÊNCIA DE CAPTURA DO CONHECIMENTO DAS SUBSIDIÁRIAS EM SUAS REDES LOCAIS

Como limitações, destacamos a falta de validação do modelo empírico. Compreendemos que o modelo conceitual necessita ser validado empiricamente, uma vez que ele se restringe a algumas variáveis. Ademais, destacamos que outras variáveis não contempladas no modelo, podem influenciar no acesso de conhecimentos e recursos da rede, tais como: sociopolíticas, institucionais, histórico e cultural. Parte-se também do pressuposto que a subsidiária detém a autonomia e mandato pela MNE, e que conflitos dentro da MNE não afetem significativamente a subsidiária em desenvolver suas estratégias e melhorar sua eficiência na obtenção de conhecimento e recursos locais. Por fim, sugerimos que estudos futuros utilizem outras bases de dados online, como Emerald Insight e Web of Science. Também sugerimos que estudos empíricos se utilizem do modelo conceitual aqui proposto, a fim de verificar se o embeddedness, o poder e a centralidade impactam de forma conjunta na aquisição ou não de recursos e conhecimentos dispostos na rede externa local, influenciando no surgimento de vantagens competitivas para subsidiária.

\section{REFERENCIAS}

ÁlVAREZ, I.; CANTWELL, J. International integration and mandates of innovative subsidiaries in Spain. Institutions and Economies, p. 415-444, 2017.

ANDERSSON, U.; DELLESTRAND, H.; PEDERSEN, T. The contribution of local environments to competence creation in multinational enterprises. Long range planning, $\mathrm{v}$. 47, n. 1-2, p. 87-99, 2014.

ANDERSSON, U.; FORSGREN, M. In search of centre of excellence: Network embeddedness and subsidiary roles in multinational corporations. Management International Review, v. 40, n. 4, 329-350, 2000.

ANDERSSON, U.; FORSGREN, M; HOLM, U. Balancing subsidiary influence in the federative MNC: A business network view. In: Knowledge, Networks and Power. Palgrave Macmillan, London, 2015. p. 393-420.

ANDERSSON, U.; FORSGREN, M.; HOLM, U. Subsidiary embeddedness and competence development in MNCs a multi-level analysis. Organization Studies, v. 22, n. 6, p. 10131034, 2001. 
BACHMANN, R.; ZAHEER, A. (Ed.). Handbook of trust research. Edward Elgar Publishing, 2006.

ANDERSSON, U.; FORSGREN M.; HOLM, U. The strategic impact of external networks: subsidiary performance and competence development in the multinational corporation. Strategic Management Journal, v. 23, n. 11, 979-996, 2002.

BATHELT, H.; MALMBERG, A.; MASKELL, P. Clusters and knowledge: local buzz, global pipelines and the process of knowledge creation. Progress in Human Geography, v. 28 n. 1, 31-56, 2006.

BONACICH, P.; ROY, W. G. Centrality, dominance, and interorganizational power in a network structure: Interlocking directorates among American railroads, 1886-1905. Journal of Mathematical Sociology, 12(2), 127-135, 1986.

BORGATTI, S. P. Centrality and network flow. Social networks, v. 27, n. 1, p. 55-71, 2005.

BOUQUET, C.; BIRKINSHAW, J. Weight versus voice: How foreign subsidiaries gain attention from corporate headquarters. Academy of Management Journal, v. 51, n. 3, 577$601,2008$.

BRESCIANI, S.; FERRARIS, A. Innovation-receiving subsidiaries and dual embeddedness: impact on business performance. Baltic Journal of Management, v. 11, n. 1, p. 108-130, 2016.

CANTWELL, J.; MUDAMBI, R. MNE competence-creating subsidiary mandates. Strategic Management Journal, v. 26, n. 12, 1109-1128, 2005.

CAPALDO, A.; GIANNOCCARO, I. Interdependence and network-level trust in supply chain networks: A computational study. Industrial Marketing Management, v. 44, p. 180$195,2015$.

CARLEY, K.M.; COLUMBUS, D. Basic Lessons in O.R.A. and AutoMap 2012. Pittsburg: Carnegie Mellon University, School of Computer Science, Institute for Software Research, Technical Report, CMU-ISR-12-107, 2012.

CASTELLS, M. Network Theory: A Network Theory of Power. International Journal of Communication, v. 5, n. 15, 2011. 
CHAE, S.; CHOI, T. Y.; HUR, D. Buyer power and supplier relationship commitment: a cognitive evaluation theory perspective. Journal of Supply Chain Management, v. 53, n. 2, p. 39-60, 2017.

CHANDLER, D.; HAUNSCHILD, P. R.; RHEE, M.; BECKMAN, C. M. The effects of firm reputation and status on interorganizational network structure. Strategic Organization, v. 11, n. 3, 217-244, 2013.

CHANG, C. W.; CHIANG, D. M.; PAI, F. Y. Cooperative strategy in supply chain networks. Industrial Marketing Management, v. 41, n. 7, 1114-1124, 2012.

CIABUSCHI, F.; DELLESTRAND, H.; HOLM, U. The role of headquarters in the contemporary MNC. Journal of International Management, v. 18, n. 3, 213-223, 2012.

CIABUSCHI, F.; HOLM, U.; MARTIN. M. O. Dual embeddedness, influence and performance of innovating subsidiaries in the multinational corporation. International Business Review, 2014.

DAS, K.; SAMANTA, S.; PAL, M. Study on centrality measures in social networks: A survey. Social Network Analysis and Mining, v. 8, n. 1, p. 13, 2018.

DJODAT, N. Network Embeddedness as a Determinant of Organizational Structure and Culture - An Empirical Study, 2016. Available at

DONG, M. C. et al. Opportunism in distribution networks: The role of network embeddedness and dependence. Production and Operations Management, v. 24, n. 10, p. 1657-1670, 2015.

DÖRRENBÄCHER, C.; GAMMELGAARD, J. Subsidiary Initiative Taking in Multinational Corporations: The Relationship between Power and Issue Selling. Organization Studies, v. 37, n. 9, 1249-1270, 2016.

DÖRRENBÄCHER, C.; GEPPERT, M.. The dark side of the moon: Power and politics in the multinational corporation. European Financial Review, 25-28, 2013.

DOZ, Y.; SANTOS J.; WILLIAMSON, P. From global to metanational: How companies win in the knowledge economy. Harvard Business School Press, Boston, Massachusetts, 2001. 
DU, J.; WILLIAMS, C. Innovative projects between MNE subsidiaries and local partners in China: exploring locations and inter-organizational trust. Journal of International Management, v. 23, n. 1, p. 16-31, 2017.

FERNER, A.; EDWARDS, T.; TEMPEL, A. Power, institutions and the cross-national transfer of employment practices in multinationals. Human relations, v. 65, n. 2, p. 163-187, 2012.

FERRARIS, A.; SANTORO, G.; DEZI, L. How MNC's subsidiaries may improve their innovative performance? The role of external sources and knowledge management capabilities. Journal of Knowledge Management, v. 21, n. 3, p. 540-552, 2017.

FRENCH, J. R.; RAVEN, B.; CARTWRIGHT, D. The bases of social power. Classics of Organization Theory, v. 7,1959.

GADDE, L. E.; HÅKANSSON, H. Professional Purchasing. London: Routledge, 1993.

GAMMELGAARD, J. Issue selling and bargaining power in intrafirm competition: the differentiating impact of the subsidiary management composition. Competition \& Change, v. 13, n. 3, 214-228, 2009.

GAMMELGAARD, J.; MCDONALD, F.; STEPHAN, A.; TÜSELMANN, H.; DÖRRENBÄCHER, C. The impact of increases in subsidiary autonomy and network relationships on performance. International Business Review, v. 21, n. 6, 1158-1172, 2012.

GASPAR, Á. Construção de redes de cooperação. Pensamento \& Realidade, v. 29, n. 2, p. $21,2014$.

GEPPERT, M.; DÖRRENBÄCHER, C. Politics and power within multinational corporations: Mainstream studies, emerging critical approaches and suggestions for future research. International Journal of Management Reviews, v. 16, n. 2, 226-244, 2014.

GIGLIO, E.; PUGLIESE, R. L.; SILVA, R. Análise dos conceitos de poder nos artigos brasileiros sobre redes. Revista de Administração da UNIMEP, v. 10, n. 3, 2012.

GOMEZ, D. et al. Centrality and power in social networks: a game theoretic approach. Mathematical Social Sciences, v. 46, n. 1, p. 27-54, 2003.

GONÇALVES, H. Manual de metodologia da pesquisa científica. Avercamp, 2005. 
GOVINDARAJAN, V.; RAMAMURTI, R. Global Strategy Journal, v. 1, n. 3-4, 191-205, 2011.

HARDY, C.; PHILlIPS, N.; CLEGG, S.. Reflexivity in Organization and Management Theory: A Study of the Production of the Research Subject'. Human Relations, v. 54, n. 5, 531-560, 2001.

HEDLUND, G. 9 Assumptions of Hierarchy and Heterarchy, with Applications to the Management of the. Organization Theory and the Multinational Corporation, p. 211, 2016.

HEIDENREICH, M. The social embeddedness of multinational companies: a literature review. Socio-Economic Review, v. 10, n. 3, p. 549-579, 2012.

HOLM, D. B.; ERIKSSON, K.; JOHANSON, J. Creating value through mutual commitment to business network relationships. Strategic management journal, v. 20, n. 5, p. 467-486, 1999.

IBARRA, H. Network centrality, power, and innovation involvement: Determinants of technical and administrative roles. Academy of Management Journal, v. 36, n. 3, 471-501, 1993.

ISAAC, V.; BORINI, F.; RAZIQ, M.; BENITO, G. From local to global innovation: The role of subsidiaries' external relational embeddedness in an emerging market. International Business Review, v. 28, n. 4, p. 638-646, 2018.

JANNECK, M.; STAAR, H. Virtual micropolitics: Informal tactics of influence and power in inter-organizational networks. In System Sciences (HICSS), 2010, 43rd Hawaii International Conference 1-10. IEEE, 2010.

KIM, D.-Y. Understanding supplier structural embeddedness: A social network perspective. Journal of Operations Management, v. 32, n. 5, p. 219-231, 2014.

KOSTOVA, T.; MARANO, V.; TALLMAN, S.. Headquarters-subsidiary relationships in MNCs: Fifty years of evolving research. Journal of World Business. v. 51, n. 1, 176-184, 2016.

LADO, A. A.; DANT, R. R.; TEKLEAB, A. G. Trust-opportunism paradox, relationalism, and performance in interfirm relationships: evidence from the retail industry. Strategic Management Journal, v. 29, n. 4, 401-423, 2008. 
LUHMANN, Niklas. Trust and power. John Wiley \& Sons, 2018.

MASCIARELLI, F.; LAURSEN, K.; PRENCIPE, A. Trapped by over-embeddedness: the effects of regional social capital on internationalization. DRUID, Copenhagen Business School, Department of Industrial Economics and Strategy/Aalborg University, Department of Business Studies, 2010.

MASKELL, P.; BATHELT, H.; MALMBERG, A.. Building global knowledge pipelines: The role of temporary clusters. European Planning Studies, v. 14, n. 8, 997-1013, 2006.

MEEHAN, J.; WRIGHT, G. H.. The origins of power in buyer-seller relationships. Industrial Marketing Management, v. 41, n. 4, 669-679, 2012.

MEO, P. et al. Using centrality measures to predict helpfulness-based reputation in trust networks. ACM Transactions on Internet Technology (TOIT), v. 17, n. 1, p. 8, 2017.

MEYER K.; MUDAMBI R.; NARULA R. Multinational enterprises and local contexts: the opportunities and challenges of multiple embeddedness. Journal of Management Studies v. 48, n. 2, 235-252, 2011.

MOLINA-MORALES, F.; MARTÍNEZ-FERNÁNDEZ, M. T. Too much love in the neighborhood can hurt: How an excess of intensity and trust in relationships may produce negative effects on firms. Strategic Management Journal, v. 30, n. 9, 1013-1023, 2009.

MORAN, P. Structural vs. relational embeddedness: Social capital and managerial performance. Strategic Management Journal, v. 26, n. 12, 1129-1151, 2005.

MORGAN, G.; KRISTENSEN, P. H. The contested space of multinationals: Varieties of institutionalism, varieties of capitalism. Human Relations, v. 59, n. 11, 1467-1490, 2006.

MUDAMBI, R.; NAVARRA, P. Is knowledge power? Knowledge flows, subsidiary power and rent-seeking within MNCs. In: The Eclectic Paradigm. Palgrave Macmillan, London, 2015. p. 157-191.

NAUDÉ, P.; BUTTLE, F. Assessing relationship quality. Industrial Marketing Management, v. 29, n. 4, 351-361, 2000. 
NICHOLLS, A.; HUYBRECHTS, B. Sustaining inter-organizational relationships across institutional logics and power asymmetries: The case of fair trade. Journal of Business Ethics, v. 135, n. 4, p. 699-714, 2016.

OLIVEIRA, L.; SACOMANO NETO, M.; BOAVENTURA, J. M. G. Influência do poder nas redes de negócio-análise da evolução da teoria. REAd. Revista Eletrônica de Administração, v. 22, n. 1, p. 1-25, 2016.

OPPER, S.; NEE, V. Network effects, cooperation and entrepreneurial innovation in China. Asian Business \& Management, v. 14, n. 4, p. 283-302, 2015.

PANTELI, N.; SOCKALINGAM, S. Trust and conflict within virtual inter-organizational alliances: a framework for facilitating knowledge sharing. Decision support systems, v. 39, n. 4, 599-617, 2005.

PARUCHURI, S. Intraorganizational networks, interorganizational networks, and the impact of central inventors: A longitudinal study of pharmaceutical firms. Organization Science, v. 21, n. 1, p. 63-80, 2010.

PERER, A.; SHNEIDERMAN, B. Balancing systematic and flexible exploration of social networks. IEEE transactions on visualization and computer graphics, v. 12, n. 5, p. 693700, 2006.

PETTICREW, M.; ROBERTS, H. Systematic reviews in the social sciences: A practical guide. John Wiley; Sons, 2008.

PIASKOWSKA, D.; TROJANOWSKI, G. Twice as smart? The importance of managers' formative-years' international experience for their international orientation and foreign acquisition decisions. British Journal of Management, v. 25, n. 1, 40-57, 2014.

PROVAN, K. G.; HUANG, K.; MILWARD, H. B. The evolution of structural embeddedness and organizational social outcomes in a centrally governed health and human services network. Journal of Public Administration Research and Theory, v. 19, n. 4, 873-893, 2009.

PROVAN, K. G.; FISH, A.; SYDOW, J. Interorganizational networks at the network level: A review of the empirical literature on whole networks. Journal of management, v. 33, n. 3, p. 479-516, 2007. 
PU, M.; SOH, P.-H. The role of dual embeddedness and organizational learning in subsidiary development. Asia Pacific Journal of Management, v. 35, n. 2, p. 373-397, 2018.

RAHIM, M. A. A Model of managerial power bases: alternative explanations of reported findings: Center for Advanced Studies in Management. In: Social Intelligence, Power, and Conflict. Routledge, 2017. p. 29-46.

RAHIM, M. A.; MAGNER, N. R.; ANTONIONI, D.; RAHMAN, S. Do justice relationships with organization-directed reactions differ across US and Bangladesh employees? International Journal of Conflict Management, v. 12, n. 4, 333-349, 2001.

REIMANN, F.; KETCHEN JR, D. J. Power in supply chain management. Journal of Supply Chain Management, v. 53, n. 2, p. 3-9, 2017.

RUGMAN, A. M. Subsidiary specific advantages and multiple embeddedness in multinational enterprises. Academy of Multinational Enterprises, 7, 1-8, 2014.

RUGMAN, A. M.; VERBEKE, A. Subsidiary specific advantages in multinational enterprises. Strategic Management Journal, v. 22, n. 3, 237-250, 2001.

SCHOEMAKER, P. J.; DAY, G. S.; SNYDER, S. A. Integrating organizational networks, weak signals, strategic radars and scenario planning. Technological Forecasting and Social Change, v. 80, n. 4, 815-824, 2013.

SHENG-YUE, H.; XU, R. Analyses of strategic alliance failure: a dynamic model. In International Conference on Management Science and Engineering-ISTP pp. 966973, 2005.

SHIJAKU, E.; LARRAZA-KINTANA, M.; URTASUN-ALONSO, A. Network centrality and organizational aspirations: A behavioral interaction in the context of international strategic alliances. Journal of International Business Studies, p. 1-16, 2018.

SOMECH, A.; DRACH-ZAHAVY, A. Relative power and influence strategy: The effects of agent/target organizational power on superiors' choices of influence strategies. Journal of Organizational Behavior, v. 23, n. 2, 167-179, 2002.

SONG, H.; EVELAND JR, W. P. The structure of communication networks matters: How network diversity, centrality, and context influence political ambivalence, participation, and knowledge. Political Communication, v. 32, n. 1, p. 83-108, 2015. 
TEECE, D.; PETERAF, M.; LEIH, S. Dynamic capabilities and organizational agility: Risk, uncertainty, and strategy in the innovation economy. California Management Review, v. 58, n. 4, 13-35, 2016.

TEIGLAND, R.; WASKO, M. Knowledge transfer in MNCs: Examining how intrinsic motivations and knowledge sourcing impact individual centrality and performance. Journal of International Management, v. 15, n. 1, 15-31, 2009.

TSAI, W. Knowledge transfer in intraorganizational networks: Effects of network position and absorptive capacity on business unit innovation and performance. Academy of management journal, v. 44, n. 5, p. 996-1004, 2001.

UZZI, B.; LANCASTER, R. Relational embeddednesss and Learning: the case of bank loan managers and their clients. Management Science. v. 49, n. 4, p. 383-399, 2003.

VAALAND, T. I.; HÅKANSSON, H. Exploring interorganizational conflict in complex projects. Industrial Marketing Management, v. 32, n. 2, 127-138, 2003.

VAHTERA, P.; BUCKLEY, P.; ALIYEV, M. Affective conflict and identification of knowledge sources in MNE teams. International Business Review, v. 26, n. 5, p. 881-895, 2017.

ZAHEER, A.; GOZUBUYUK, R.; MILANOV, H. It's the connections: The network perspective in interorganizational research. Academy of Management Perspectives, v. 24, n. 1, 62-77, 2010.

ZANDER, I.; MATHEWS, J. A. Beyond heterarchy: Emerging futures of the hypermodern MNC. In U. Andersson, U. Holm (Eds.), Managing the Contemporary Multinational: The Role of Headquarters, 33-59. Cheltenham: Edward Elgar Publishing, 2010. 